\title{
Pseudocholinesterase Deficiency and Patient Perspectives
}

\author{
Jevaughn Davis, $M^{1^{*}}$, Anita Vincent, $M D^{2}$, and Geetha Shanmugam, $M D^{3}$
}

${ }^{1}$ Resident Physician, Department of Anesthesiology and Critical Care, George Washington School of Medicine and Health Sciences, Washington DC, USA

${ }^{2}$ Attending Physician, Assistant Professor, Section Chief-Trauma Anesthesiology and Director of Resident Education, Neuroanesthesiology, Department of Anesthesiology and Critical Care, George Washington School of Medicine and Health Sciences, Washington DC, USA

${ }^{3}$ Attending Physician, Assistant Professor, Department of Anesthesiology and Critical Care, George Washington School of Medicine and Health Sciences, Washington DC, USA

*Corresponding author: Dr. Jevaughn Davis, Resident Physician, Department of Anesthesiology and Critical Care, George Washington School of Medicine and Health Sciences, $90023^{\text {rd }}$ St NW, Washington DC 20037, USA

\begin{abstract}
Pseudocholinesterase deficiency, commonly referred to as Butyrylcholinesterase deficiency, is a rare, inherited, or acquired condition that results in decreased or absent enzymatic activity. Pseudocholinesterase is a plasma enzyme that is responsible for the breakdown of succinylcholine and mivacurium, which are commonly used paralytic agents. People with this condition are usually unaware they have the disease, and the disease may not manifest until extubation at the end of surgery. These patients will usually have abnormally prolonged paralysis time and require mechanical ventilation well into the post anesthesia care unit because of failure to meet qualitative and quantitative extubation criteria. Treatment is mainly supportive with continued mechanical ventilation until return of muscle function because paralysis can last from minutes to hours and re-sedation to limit patient discomfort. We present two cases of prolonged paralysis after succinylcholine administration subsequently confirmed by laboratory testing to be pseudocholinesterase deficiency.
\end{abstract}

\section{Keywords}

Factors causing prolonged neuromuscular blockade, Suxamethonium, Contra-indications, Pseudocholinesterase synthesis, Butyrylcholinesterase, deficiency

\section{Introduction}

Emergence from anesthesia can be delayed by many factors; one of the more rare causes is pseudocholinesterase deficiency. This disorder is marked by a decrease or absence of the pseudocholinesterase enzyme. This enzyme is a product of liver synthesis and aids in the degradation and hydrolysis of anesthetic esters including muscle relaxants such as succinylcholine and mivacurium. This enzyme also aides in the degradation of ester-derived local anesthetic agents such as procaine, tetracaine and cocaine. Succinylcholine and less commonly, mivacurium, are used to aid in endotracheal intubation and to keep patients paralyzed for surgery. The half-life of succinylcholine is approximately 5 to 10 minutes, while mivacurium's half-life is about 1.5 to 4 minutes. Pseudocholinesterase deficiency is of importance to anesthesiologists because patients with this deficiency will exhibit prolonged paralysis with the aforementioned agents leading to continued mechanical ventilation hours after surgical completion. In a literature review consisting of 40 case reports, Hackett and Sakai found the length of paralysis ranged from 50 minutes to 10 hours [1].

This disease can be inherited or acquired. Pseudocholinesterase deficiency is inherited in an autosomal recessive manner, and affected individuals inherit mutations on the butyrylcholinesterase gene, which is located on chromosome $3[1,2]$. Heterozygotes inherit one copy of the defective gene while homozygotes inherit both defective genes. Acquired causes of this deficiency include, but are not limited to: liver failure, renal failure, massive burns, malignancy, hypothermia, 
hypothyroidism, extremes of age, hypoalbuminemia/ malnutrition, pregnancy, and HELLP syndrome [15]. Iatrogenic etiologies include organophosphates, cyclophosphamide, diethylstilbestrol, MAO inhibitors, phenelzine, and alkylating agents [1-5]. Incidence of disease differs among genotypes. The incidence among homozygotes is 1 per 2000-5000 persons and is 1 per 500 among heterozygotes; within this incidence, men have a 2:1 prevalence when compared to women $[2,5]$.

\section{Report}

\section{Case 1}

A 40-year-old woman presented for dilation and evacuation. Past medical history includes acid reflux and being a former smoker. She had no allergies and was not taking any medications. Family history was noncontributory. The patient denied previous surgeries and had never received an anesthetic. Physical exam was unremarkable, and ASA was 2.

After being premedicated with $2 \mathrm{mg}$ Midazolam and preoxygenated, general anesthesia was induced with $100 \mathrm{mg}$ lidocaine, $200 \mathrm{mg}$ Propofol and $100 \mathrm{mg}$ Succinylcholine for endotracheal intubation. Anesthesia was maintained with $50 \%$ oxygen and sevoflurane. Initial vitals were normal. The surgery lasted 45 minutes. Intraoperative medications were $50 \mathrm{mcg}$ fentanyl, 10 units oxytocin, $8 \mathrm{mg}$ dexamethasone and $4 \mathrm{mg}$ ondansetron. At the end of the procedure, the patient demonstrated no respiratory effort. Based on the medical research council's (MRC) muscle grading system, the patient had a score of 0 which was suggestive of paralysis. Neuromuscular train-offour ratio was 0 out of 4 , indicating full paralysis. By this time, the inhalational agent had been washed out. The patient was re-sedated. Mechanical ventilation was continued, and the patient was transferred to the post anesthesia care unit (PACU) for recovery. In the PACU, vitals were normal. Neuromuscular train-offour ratio was continuously monitored. Within 1 hour, the patient began to move weakly but was unable to follow commands. After another 1.5 hours, the patient demonstrated 4/4 muscle strength and MRC score increased from 0 to 5 . The patient was extubated to nasal canula. The total time for recovery after succinylcholine administration was 210 minutes.

To confirm the suspected diagnosis of pseudocholinesterase deficiency, a dibucaine test was sent and confirmed that the patient had a reduced dibucaine number of 34 . Succinylcholine was added to her medical record as an adverse drug reaction.

\section{Case 2}

A 38-year-old woman presented for hysteroscopy and myomectomy. Past medical history includes exercise induced asthma, fibroids and headaches. Family history was noncontributory. The patient was a social drinker and former smoker. Physical exam was unremarkable. ASA was a 2.

The patient was premedicated with $2 \mathrm{mg}$ Midazolam and pre-oxygenated. General anesthesia was induced with $100 \mathrm{mg}$ lidocaine, $200 \mathrm{mg}$ Propofol. LMA was attempted but could not be positioned well so she was given $100 \mathrm{mg}$ IV Succinylcholine for uncomplicated endotracheal intubation. Anesthesia was maintained with $70 \%$ oxygen and desflurane. Initial vitals were normal. The surgery lasted 30 minutes. Additionally, she was given $4 \mathrm{mg}$ Dexamethasone, $4 \mathrm{mg}$ Ondansetron, $50 \mathrm{mg}$ Ketamine and $30 \mathrm{mg}$ Ketorolac. At the end of the case, the patient was globally weak. She had a MRC score of 0 with no visible muscle contraction. Neuromuscular train-of-four ratio revealed 0 out of 4 twitches. She was re-sedated with propofol then transferred to the PACU where mechanical ventilation was continued. Neuromuscular monitoring was continued. After 360 minutes, the patient was successfully extubated after repeated train-of-four ratio revealed 4 out of 4 twitches and MRC muscle score of 5 .

A dibucaine number confirmed the suspected diagnosis and revealed a dibucaine number of 44 , which indicated heterozygosity for pseudocholinesterase deficiency.

\section{Patient Perspective}

\section{Case 1}

"I could tell something was wrong. I vaguely remember what happened. I felt trapped within my body. I could hear people speaking to me, but I couldn't respond. I couldn't open my mouth or move my arms or legs. I felt extremely weak. The last thing, I remember was all the doctors around me and then everything felt like a dream until I woke up in the recovery bay. I was terrified during the incident. The doctors explained what happened and how this can be prevented in the future. Multiple doctors also followed up multiple times after I left the hospital which was reassuring. My children and siblings were tested so at least they can avoid a situation like this since I now know this disorder is genetic."

\section{Case 2}

"I was aware something bad was occurring. I felt an enormous amount of panic and doom, people were talking, and I couldn't move anything, I could only look up. It felt like I was locked into place. It was a very disturbing and surreal feeling. When I eventually returned to normal, the doctors and staff were very calming and supportive. One year later and I can still vividly remember the sensation of feeling so extremely weak and powerless, it was quite disturbing but since then I have had great follow up and education on this disease. My twin sister recently tested positive for the deficiency too, I believe she was also heterozygous. Eventually I want to have my children tested." 


\section{Discussion}

Pseudocholinesterase deficiency is usually diagnosed retrospectively after a patient has received general anesthesia with succinylcholine or mivacurium used for endotracheal intubation. The differential diagnosis for pseudocholinesterase deficiency includes many other causes of delayed extubation such as narcotic overdose, hypermagnesemia, hypokalemia, hypophosphatemia, cholinergic crisis, myasthenia crisis and residual diaphragmatic blockade. The anesthetic record if medications are documented correctly will quickly rule in or out narcotic overdose. Pinpoint pupils will reveal narcotic overdose and giving naloxone reverses the issue. A thorough history and physical exam would give an inclination as to whether the patient carried a diagnosis of Myasthenia gravis. Preoperative labs, if obtained, will identify electrolyte abnormalities such as hypermagnesemia, hypokalemia, and hypophosphatemia. Post procedure labs can help to rule out electrolyte disturbances as causes of this disorder. In both the cases presented above, peripheral nerve monitoring revealed $0 / 4$ twitches demonstrating lasting paralysis, which led to the suspected diagnosis of pseudocholinesterase deficiency. A subsequent dibucaine number confirmed the diagnosis. Dibucaine number indicates zygosity and genetic origin of disease. Dibucaine is a local anesthetic agent that inhibits the enzymaticactivity of pseudocholinesterase; the lower the quantity of dibucaine, the lower the absolute inhibition of dibucaine and therefore the lower the dibucaine number. Numbers less than 30 indicate homozygous pseudocholinesterase deficiency, numbers 30-70 indicate heterozygosity for the mutation, and numbers greater than 70 indicate normal pseudocholinesterase activity [1]. Patient 1 had a dibucaine number of 34 , and patient 2 had a number of 44, which indicate both were heterozygous for the mutation. Duration of blockade is linked to zygosity. Heterozygotes present with a $30 \%$ increase in the duration of blockade, in minutes to hours. Homozygotes exhibit an average of at least 2 hours of prolonged blockade [1,2].

The mainstay of treatment is continued mechanical ventilation with sedation and continued peripheral nerve monitoring until paralysis ends and spontaneity of muscle activity returns [1-2,4-6]. It has been theorized that fresh frozen plasma (FFPs) or packed red blood cells ( $p R B C s$ ) can be administered to shorten the duration of the prolonged block $[1,2,4]$. When FFPs or pRBCs are given, the patient is being provided with exogenous pseudocholinesterase. This is rarely done because there is less risk associated with continued mechanical ventilation for a few extra hours compared with giving blood products. In fact, Hackett and Sakai found via a literature review that of the reported 40 cases from 1956 to 2011, there has only been one reported case where FFPs were used [1]. Reluctance to use FFPs is not only due to the risk versus benefit assessment, but also due in part to the unreliability of transfusions in blockade reversal [1,2]. Additionally, there have been no case reports of long-term sequelae or mortality from pseudocholinesterase deficiency. Pharmacological blockade reversal is also not advised; agents such as neostigmine and physostigmine, which are commonly used anesthetic agents, which antagonize and inhibit pseudocholinesterase activity. Attempted reversal with these agents will prolong the neuromuscular blockade.

To prevent future incidence of prolonged paralysis, patients with pseudocholinesterase deficiency should avoid succinylcholine and mivacurium. This involves properly educating patients on this new diagnosis. Another failsafe method to prevent reoccurrence is to list mivacurium and succinylcholine as sources of patient allergies or adverse reactions. Reoccurrence can also be deterred by a thoroughly conducted preoperative assessment by the anesthesia provider. Questions aimed at previous surgeries and issues with anesthesia in the past can elicit a history of pseudocholinesterase deficiency from patients [6]. Once confirmed, providers can adjust anesthetic plans to avoid medications that would cause prolong blockade and difficulties with extubation. Fortunately, there are other paralytic alternatives that have different metabolism mechanisms, such as rocuronium, vecuronium, atracurium and cisatracurium that will not significantly prolong neuromuscular blockade.

From the patient perspective provided above, prolongedintubationsecondarytopseudocholinesterase deficiency can be quite distressing to patients due to interruption of sedation when attempting to extubate. It is imperative that patients are re-sedated in a timely manner to limit patient discomfort. Efforts should be focused on not interrupting sedation. Ways to decrease patient discomfort and stress should be aimed at limiting interruption of sedation until train of four is obtained towards the end of a procedure after succinylcholine is given. While succinylcholine is relatively reliable in its metabolism, given the risk and emotional distress to this patient population, perhaps neuromuscular monitoring should be employed some time before attempts at extubation.

Pseudocholinesterase deficiency is inherited in an autosomal recessive manner; however, given the large genetic component, it is recommended that immediate family members get tested. Testing can be done with a dibucaine number as mentioned above, or providers can send for a plasma pseudocholinesterase level.

\section{Acknowledgements}

This case report was written and published with the written consent of the patients.

\section{Author Contributions}

J.D., A.V., and G.S. conceived the idea of the case 
report. G.S. and A.V. identified and managed case 1 and 2 respectively. J.D. is the guarantor. J.D. performed the literature review. J.D. wrote the original draft; A.V., and G.S. were responsible for reviewing, editing, and rewriting.

\section{Conflict of Interests}

All authors have completed the ICMJE uniform disclosure form. The authors have no conflicts of interest to declare.

\section{References}

1. Hackett PJ, Sakai T (2012) Pseudocholinesterase Deficiency: A Case Report and Literature Review. Open Journal of Anesthesiology 2: 188-194.
2. Trujillo R, West WP (2021) Pseudocholinesterase Deficiency. In: StatPearls Treasure Island, FL: Stat Pearls Publishing.

3. Andersson ML, Moller AM, Wildgaard K (2019) Butyrylcholinesterase deficiency and its clinical importance in anaesthesia: A systematic review. Anaesthesia 74: 518528.

4. Zhang C, Cao H, Wan ZG, Wang J (2018) Prolonged neuromuscular block associated with cholinesterase deficiency. Medicine 97: e13714.

5. Zencirci B (2009) Pseudocholinesterase enzyme deficiency: A case series and review of the literature. Cases Journal 2.

6. Lee S, Han JW, Kim ES (2013) Butyrylcholinesterase deficiency identified by preoperative patient interview. Korean J Anesthesiol 65: S1-S3. 